\title{
OCT Angiography: A New Multi Modal Imaging in CNVM
}

\author{
Shah Md Rajibul Islam* \\ Classified Eye Specialist, Military Hospital, Bangladesh
}

*Corresponding author: Shah Md Rajibul Islam, Classified Eye Specialist, Dhaka, Bangladesh.

Received Date: October 28, 2019

Published Date: November 12, 2019

\section{Short Communication}

Choroidal neovascularization is a manifestation of the diseases affecting choroid, Bruch's membrane and retinal pigment epithelium (RPE). Choroidal neovascular membrane (CNVM) consists of an abnormal growth of vessels originating from the choroid, located initially within the layers of Bruch's membrane; subsequently it may penetrate the RPE and grow under neurosensory retina. CNVM in the macular area results in severe visual loss [1].

The pathogenesis of CNV is multifactorial. Alterations in Bruch's membrane, migration of macrophages and production of vascular endothelial growth factor (VEGF), play an important role in the development of this disease. CNVM may occur as an idiopathic entity or in association with number of pathological conditions like age-related macular degeneration (ARMD), pathologic myopia, angioid streaks, choroidal tears, in certain inflammatory diseases of choroid and retina, among others [1,2].

Histologically, neovascular membranes are classified into:

- $\quad$ Type 1 when the neovascular membrane is located below the RPE.

- $\quad$ Type 2 passes through the RPE and is located above the RPE in the subretinal space.

- $\quad$ Type 3 also known as Retinal Angiomatous Proliferation (RAP), corresponds to neovascularization that develops within the neurosensory retina [3].

Type $1 \mathrm{CNV}$ is observed by OCT-A as a neovascular coralliform complex with afferent vessel, originating in the choroid. Type $2 \mathrm{CNV}$ is visualized as a neovascular network that grows from the choroid vasculature, traverses the RPE-Bruch's membrane complex into the subretinal space. Type $3 \mathrm{CNV}$ is clinically seen as tiny intra- and subretinal hemorrhages that correlate on OCT-A to an intraretinal anastomosis originating in the deep capillary plexus of the retina [4].

Any disease that results in an abnormality at the level of the RPE-Bruch's membrane-choriocapillary complex, can be associated with CNVM. The incidence of CNVM in pathological myopia is approximately $5 \%$ [5]. The reported incidence of CNVM is $60 \%$ to $86 \%$ in eyes with angioid streaks [6]. CNVM in the macular area is a major cause of visual loss in presumed ocular histoplasmosis syndrome (POHS). CNVM occurs in other inflammatory conditions like multifocal choroiditis, serpiginous choroiditis and certain other inflammatory diseases [1]. When CNVM develops in the absence of atrophic chorioretinal scar, drusen or another retinal abnormality, it is termed idiopathic [1].

Traumatic ruptures of the Bruch's membrane are commonly seen following severe contusion injuries to the eye. Late loss of central vision following trauma can occur due to development of CNVM. Choroidal osteoma is a benign ossifying tumour of the eye which is often associated with CNVM [1].

Age related macular degeneration (ARMD) is an ageing change within the macula at around 50 years and older individuals [7]. Age related changes in the Bruch's membrane and drusen formation is the primary pathogenesis [8]. ARMD is the third leading cause of blindness following cataract and glaucoma, and the wet variety, responsible for $90 \%$ of visual loss, is characterized by the presence of CNVM [9].

The classification of CNVM is based on the extension of abnormal neovascularization [10]. Fundus fluorescein angiogram (FFA) is the current standard investigation to diagnose and monitor disease progression [11]. But FFA also has some disadvantages. There are contraindications of its use as well as adverse effects 
including nausea, vomiting, and anaphylactic shock, even death [12].

In 2015, Optical Coherence Tomography Angiography (OCT A) became available for clinical use. Since then features of diabetic retinopathy, choroidal neovascularization, macular telangiectasia, vascular occlusion has been characterized by OCT A. OCT A is a noninvasive imaging technique that uses motion contrast to create high resolution depth resolved angiographic images of vascular flow [11]. The software compares the phase variance between consecutive OCT B scans acquired at the same retinal cross section to detect motion contrast. Differences represent movement of red blood cells within the vessels. From multiple retinal cross sections, a retinal map can be constructed. This allows simultaneous evaluation of retinal blood flow pattern and structural information. OCT angiograms can be segmented into different layers to evaluate different vascular layer separately [13,14]. OCT A imaging also enables quantitative analysis of neovascular complexes over time.

The limitations of OCT A are that image resolution is reduced when scanning area is increased. This can be improved by montaging multiple smaller scans together. Multiple OCT A image artifacts can affect the image quality. Motion artifacts due to blinking or gross eye movement appear as black and white lines vertically and horizontally. Motion correction software can result in vessel doubling, stretching of image and loss of detail. Signal attenuation can occur. Automated software (can remove these artifacts) [15].

The short acquisition time and noninvasive nature of OCTA may provide a practical modality for sequential imaging in patients with CNVM [16].

\section{Acknowledgement}

None.

\section{Conflict of Interest}

No Conflict of interest.

\section{References}

1. Bhatt NS, Diamond JG, Jalali S, Das T (1998) Choroidal Neovascular Membrane. Indian J Ophthalmology 46(2): 67-80.
2. Carnevali A, Cicinelli MV, Capuano V, Corvi F, Mazzaferro A, et al. (2016) Optical Coherence Tomography Angiography: A Useful Tool for Diagnosis of Treatment-Naïve Quiescent Choroidal Neovascularization. Am J Ophthalmol 169: 189-198.

3. Jia Y, Bailey ST, Wilson DJ, Tan O, Klein ML, et al. (2014) Quantitative optical coherence tomography angiography of choroidal neovascularization in age-related macular degeneration. Ophthalmology 121(7): 1435-1444.

4. Talisa E de Carlo, Caroline R Baumal (2016) Advances in Optical Coherence TomographyAngiography. US Ophthalmic Review 9(1): 3740.

5. Grossniklaus HE, Green WR (1992) Pathologic findings in pathologic myopia. Retina 12(2): 127-133.

6. Piro PA, Scheraga D, Fine SL, Angiod streaks (1983) Natural history and visual prognosis. In: Fine SL, Owens S (eds), Management of Retinal Vascular and Macular Disorders, USA, Pp. 136-39.

7. Bird A, Frederick L, Wilkinson CP, Chakravarthy U, Chew E, et al. (2013) Clinical classification of age-related macular degeneration. Ophthalmology 120(4): 844-851.

8. Reynolds R, Seddon JM, Yu Y, Daly MJ, Rosner B (2011) Risk models for progression to advanced age-related macular degeneration using demographic, environmental, genetic and ocular factors. Ophthalmology 118(11): 2203-2211.

9. Mariotti SP, Pascolini D (2012) Global estimates of visual impairment. Br J Ophthalmol 96(5): 614-618.

10. Cook HL, Patel PG, Tufail A (2008) Age-related macular degeneration: diagnosis and treatment. British Medical Bulletein 85: 127-149.

11. Jennifer I (2013) Age-related macular degeneration. ( $3^{\text {rd }}$ edn), CRC Press.

12. Xu K, Tzankova V, Li C, Sharma (2016) Intravenous fluorescein angiography associated adverse reactions. Can J Ophthalmol 51(5): 321325.

13. Mastropasqua R, Di Antonio L, Di Staso S, Agnifili L, Di Gregorio A et al. (2015) Optical Coherence Tomography Angiography in Retinal Vascular Disease and Choroidal Neovascularization. J Ophthalmol.

14. Kim DY, Fingler J, Zawadzki RJ, Park SS, Morse LS, et al. (2013) Optical Imaging of the Chorio-retinal Vasculture in the Living Human Eye. Proc Natl Acad Sci USA $110(35)$ :14354-14359.

15. Schwartz DM, Fingler J, Kim DY, Zawadzki RJ, Morse LS, et al. (2014) Phase Variance Optical Coherence Tomography. Ophthalmology 121(1) :180-187.

16. David R Chow (2018) Paulo Ricardo Chaves de Oliveira OCT Angiography. Thieme Medical Publishers, USA. 